\title{
Impurity conundrum of organic room temperature afterglow
}

Chengjian Chen ${ }^{1}$, Zhenguo $\mathrm{Chi}^{2}$, Kok Chan Chong ${ }^{1}$, Andrei S. Batsanov ${ }^{3}$, Zhan Yang ${ }^{2}$, Zhu $\mathrm{MaO}^{2}$, Zhiyong Yang ${ }^{2} \& \mathrm{Bin} \mathrm{Liu}^{1 *}$

${ }^{1}$ Department of Chemical and Biomolecular Engineering, National University of Singapore, 4

Engineering Drive 4, Singapore 117585, Singapore.

${ }^{2}$ School of Chemistry, Sun Yat-sen University, Guangzhou 510275, China.

${ }^{3}$ Department of Chemistry, Durham University, Durham DH1 3LE, UK.

Commercial carbazole has been widely used to synthesize organic functional materials that entwine with the recent breakthroughs in thermally activated delayed fluorescence ${ }^{1,2}$, organic luminescent radicals $^{3}$ and organic laser diodes ${ }^{4}$. Recently, the strategy of stabilizing triplet excited states in carbazole derivatives ignited the booming development of organic room temperature afterglow (RTA) ${ }^{5}$. The unusual RTA of carbazole and its derivatives was elaborated by crystal quality and packing ${ }^{5,6}$. However, impurity hypotheses in organic RTA have been under debate for nearly a century ${ }^{7}$. Here we show that an isomer of carbazole, accompanying the commercial sources with less than $0.5 \%$, is the key to activating RTA for many carbazole derivatives. As compared to commercial carbazole, the fluorescence of lab-synthesized carbazole is blue-shifted by $54 \mathbf{~ n m}$ and the well-known RTA $^{6}$ disappears. The same phenomenon is also observed for a series of carbazole derivatives $^{5,8}$. Interestingly, even $0.01 \%$ isomer doping could yield the reported RTA $^{6}$. Our results demonstrate that the isomer doping in carbazole derivatives is responsible for their RTA. The impurity effect has also been confirmed for dibenzothiophene based RTA. We anticipate that isomer doping effect is applicable to many organic semiconductors derived from commercial carbazole, which will drive the review of organic functional materials in optoelectronics ${ }^{1,5,9}$. 
Bologna Stone was the first scientifically documented afterglow material. After some 400 years of studies, it was proven that the afterglow was due to impurities ${ }^{10}$. Two decades ago, the research interest of inorganic afterglow was heated up by the $\mathrm{SrAl}_{2} \mathrm{O}_{4}: \mathrm{Eu}^{2+}, \mathrm{Dy}^{3+}$ doping system $^{11}$. Nowadays, inorganic afterglow has been extensively employed to produce luminous paints, dials, emergency signs and so on. As compared to the inorganic counterpart, organic afterglow materials show more advantages, such as flexibility, transparency, solubility and color tunability ${ }^{12-14}$. Organic afterglow materials including carbazole, dibenzothiophene, dibenzofuran, fluorene, benzoin and their derivatives have been successfully developed ${ }^{5-8,12}$. Meanwhile, their impurity hypotheses have been under debate since the early $20^{\text {th }}$ century 6,7 , but without solid evidence. For example, through sublimation and recrystallization, Clapp still claimed that small traces of impurities should be considered in $1939^{7}$, while Bilen ruled out the impurity effect in $1978^{6}$. Very recently, Adachi and Kabe developed the TMP/PPT ${ }^{12}$ organic system, of which the afterglow lasted for more than $1 \mathrm{~h}$ at $300 \mathrm{~K}$. Tang et al. also reported the TPP-3C2B:DMA ${ }^{15}$ organic system with up to $7 \mathrm{~h}$ detectable RTA, which implies that the doping system is of significance. Notably, the phosphorescence of many solid organic compounds was attributed to small traces of impurities ${ }^{7,16}$. Therefore, one feasible solution to impurity conundrums can open the door to various organic doping systems.

In 1872, Craebe and Glazer isolated carbazole $(\mathrm{Cz})$ from the anthracene fraction of coal tar (Extended Data Fig. 1), which is the current commercial source for $\mathrm{Cz}^{17}$. During the recent 5 years, over 5600 publications containing $\mathrm{Cz}$ derivatives were published. Some of these carbazole derivatives have directly led to the recent breakthroughs, such as thermally activated delayed fluorescent light-emitting diodes ${ }^{1,2}$, radical-based light-emitting diodes ${ }^{3}$, and organic laser diodes ${ }^{4}$. In particular, the commercial $\mathrm{Cz}$ derivatives are the current focus of singlecomponent organic RTA studies ${ }^{5,8,18-25}$. However, some fundamental inconsistencies emerged when the same compounds were reported by different groups ${ }^{8,16,20-22}$. We thus examined the 
commercial Cz from Tokyo Chemical Industry (TCI), J\&K, Sigma-Aldrich, Aladdin, and all of them showed RTA after recrystallization ${ }^{6}$, but with varied intensities and durations.

Hence, we synthesized $\mathrm{Cz}$ (Lab-Cz) from 2-aminobiphenyl. Surprisingly, the fluorescence of Lab-Cz is blue-shifted by $54 \mathrm{~nm}$ (Extended Data Fig. 2) and the well-known RTA disappears as compared to the $\mathrm{Cz}$ from TCI (TCI-Cz) in the same crystal state (Fig. 1a) ${ }^{20}$. This result indicates that some impurities play a key role. Due to the extremely small traces and lack of available solutions, many methods had been tried without success until high-performance liquid chromatography (HPLC) was rationalized to monitor the onset absorption at $346 \mathrm{~nm}$. This is essential because when monitored at $294 \mathrm{~nm}$, the information of impurity is easily covered by the maximum absorption of the dominant $\mathrm{Cz}$ (Extended Data Fig. 3). However, at the onset absorption of $346 \mathrm{~nm}$, the impurity peak is uncovered gradually when optimizing acetonitrile-water ratio from $95 / 5$ to $50 / 50$ (v/v, Fig. 1b). After isolating $\sim 10 \mathrm{mg}$ of the impurity from $100 \mathrm{~g}$ of TCI-Cz, X-ray crystallography revealed its structure as an isomer of carbazole (Cc, Fig. 1b and Extended Data Fig. 4a). 


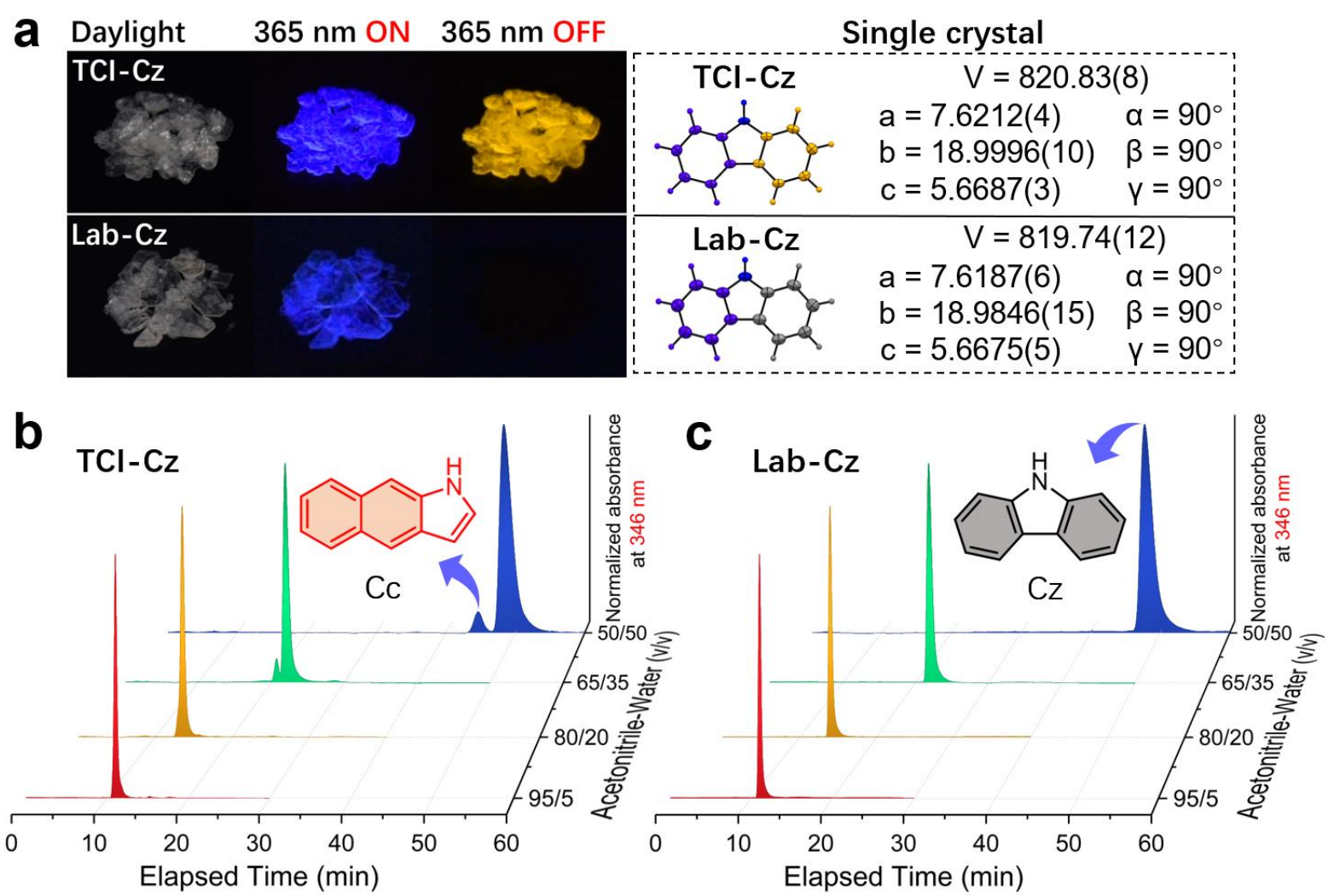

Fig. 1 | Investigations of carbazole afterglow paradox. a, Photographs of TCI-Cz and Lab$\mathrm{Cz}$ crystals at daylight, $365 \mathrm{~nm}$ irradiation ON/OFF, and their single crystal structures with unit cell parameters. b, c, HPLC spectra of TCI-Cz (b) and $\mathrm{Lab}-\mathrm{Cz}$ (c) monitored at the onset absorption of $346 \mathrm{~nm}$ with $95 / 5$ to $50 / 50$ acetonitrile (ACN)-water ratio (v/v), and chemical structures of $\mathrm{Cc}$ and $\mathrm{Cz}$.

We further studied commercial $\mathrm{Cz}$ from J\&K, Sigma-Aldrich, Aladdin and identified the same impurity, but with different content (Fig. 2a). This widespread isomer in commercial sources may affect a wide variety of organic semiconductors derived from commercial $\mathrm{Cz}$, as the isomer $\mathrm{Cc}$ inherits similar reactivity. We therefore take $\mathrm{CPhCz}$ and $\mathrm{DPhCzT}$ (Fig. 2c) as the examples to investigate the contribution of impurities to their reported ultralong phosphorescence (also called afterglow $)^{5,8}$.

Having revealed $\mathrm{Cc}$ in commercial $\mathrm{Cz}, \mathrm{TCI}-\mathrm{CPhCz}$ (synthesized from $\mathrm{TCI}-\mathrm{Cz}$ ) was purified by column three times before recrystallization (details in Methods). In the same single crystal state, the carefully purified TCI-CPhCz still exclusively shows RTA in contrast to $\mathrm{Lab}-\mathrm{CPhCz}$ (synthesized from Lab-Cz, Fig. 2d) ${ }^{8}$. Meanwhile, the optimized HPLC reveals that there is a 
small trace of impurity in TCI-CPhCz upon monitoring at the onset absorption of $354 \mathrm{~nm}$, and it is later quantified to be $0.1 \%$ with a reference (Fig. 2b). Analogously, $\sim 22 \mathrm{mg}$ of the impurity was isolated and X-ray crystallography identified its structure as $\mathrm{CPhCc}$ (Extended Data Fig. $4 \mathrm{~b}$ and Fig. 2c). We confirmed that $\mathrm{CPhCc}$ could be easily generated within TCI-CPhCz, after using the synthetic condition of $\mathrm{CPhCz}$ to efficiently scale up $\mathrm{CPhCc}$ from $\mathrm{Cc}^{8}$. Meanwhile, using the same condition as that of $\mathrm{DPhCzT}^{5}$, DPhCcT (Fig. 2c) was synthesized from Cc. To explore the generality, another 8 representative RTA $\mathrm{Cz}$ derivatives were further tested (Extended Data Fig. 5) 5,8,16,20-24. It was found that their reported RTA was only observed with the crystals synthesized from TCI-Cz, but not from Lab-Cz. Taken together, we propose that the isomer $\mathrm{Cc}$ in commercial $\mathrm{Cz}$ is responsible for their RTA. This result also indicates that the presence of $\mathrm{Cc}$ should be taken into consideration for other organic semiconductors derived from commercial $\mathrm{Cz}$, especially for those being used in optoelectronic applications ${ }^{1-4,9}$. Moreover, the afterglow of $0.01 \% \mathrm{Cc} / \mathrm{Cz}$ (Extended Data Fig. 6b) implies that this isomer doping is extremely effective. The ultra-low content explains why the impurity effect has been largely ignored ${ }^{5,6,8,18-25}$. 
a

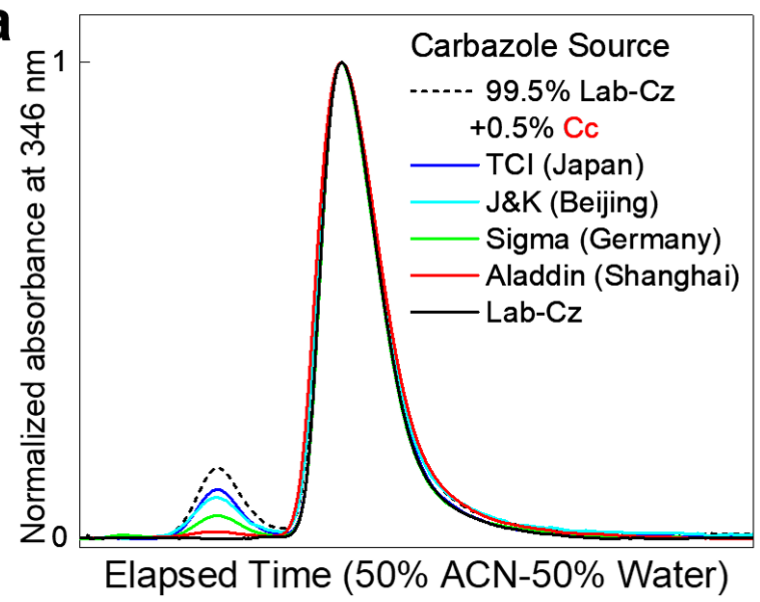

C

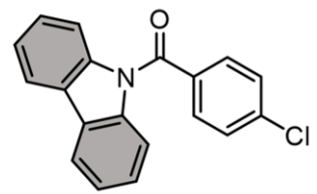

$\mathrm{CPhCz}$

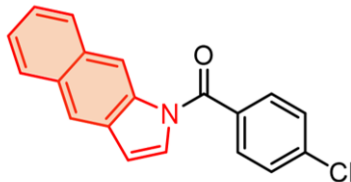

$\mathrm{CPhCc}$
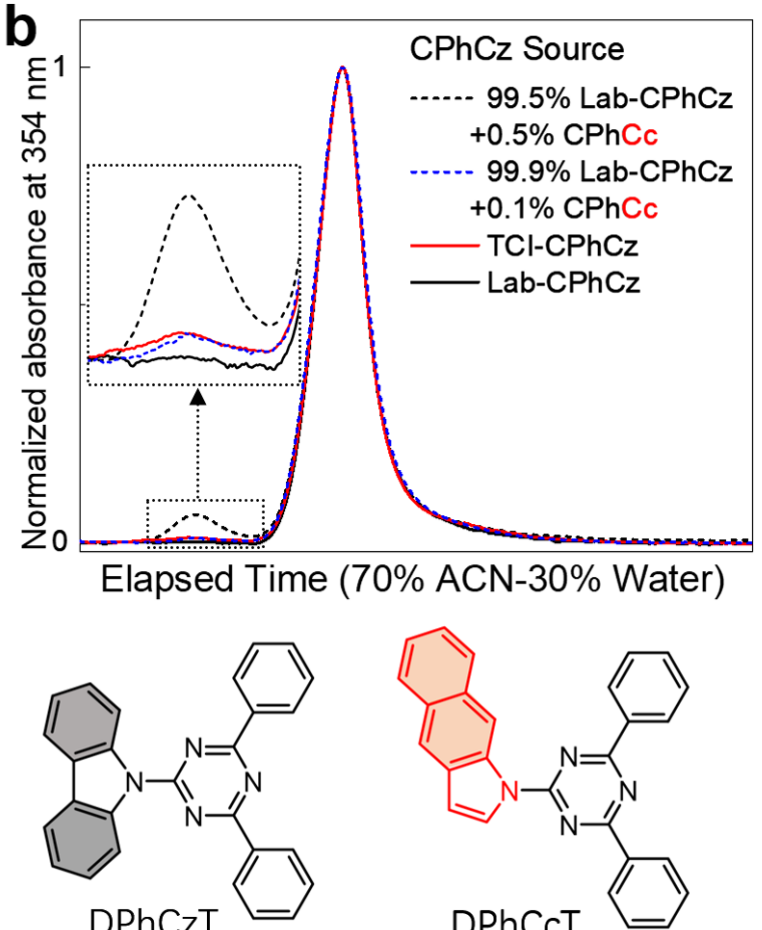

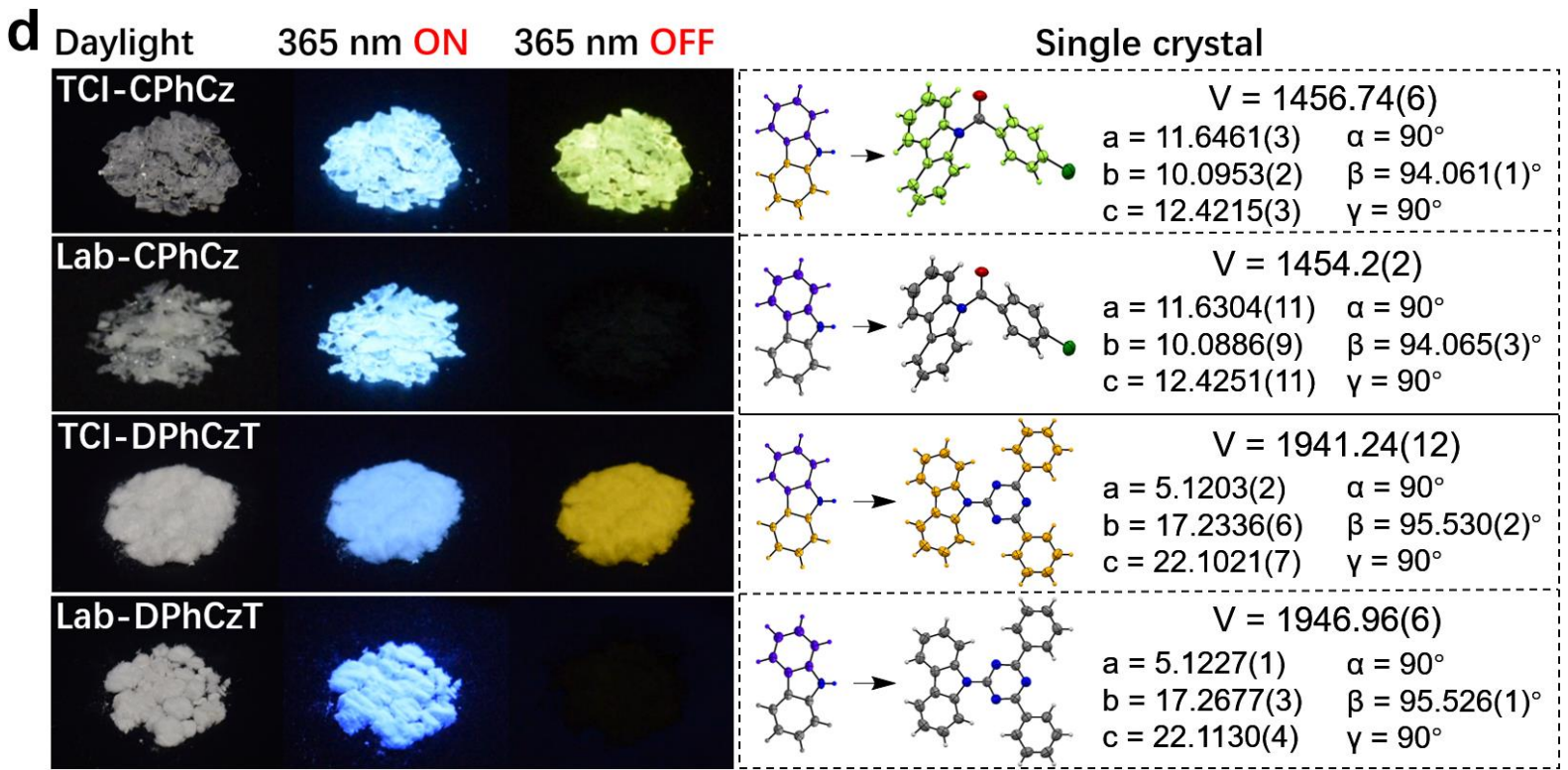

Fig. 2 Isomer doping effect of carbazole derivatives. a, b, HPLC spectra monitored at the onset absorption of $346 \mathrm{~nm}$ for $\mathrm{Cz}$ (a) and $354 \mathrm{~nm}$ for $\mathrm{CPhCz}$ (b) from commercial and labsynthesized sources, the elapsed time aberrations caused by injections were shifted by setting $\mathrm{Lab}-\mathrm{Cz}$ and $\mathrm{Lab}-\mathrm{CPhCz}$ as the reference, respectively. c, Chemical structures of $\mathrm{CPhCz}$, CPhCc, DPhCzT and DPhCcT. d, Photographs of $\mathrm{CPhCz}$ and DPhCzT crystals at daylight, $365 \mathrm{~nm}$ irradiation ON/OFF, and their single crystal structures with unit cell parameters. To distinguish, TCI or Lab was respectively added in front of $\mathrm{CPhCz}$ and $\mathrm{DPhCzT}$ according to their syntheses from TCI-Cz or Lab-Cz, i.e., TCI-CPhCz, Lab-CPhCz, TCI-DPhCzT and Lab$\mathrm{DPhCzT}$. All the \% for doping systems refer to mol\% in this paper. 
RTA based on the isomer doping differs from most design paradigms ${ }^{5,12,15,21,24-26}$. The very similar molecular structure and size (Extended Data Fig. 7a) allow the isomers to interact tightly $^{26}$, meanwhile, generating defects by the tiny difference. To understand how the isomer affects RTA, photoluminescence (PL) characteristics were investigated with $0 \%, 0.5 \%, 1 \%$, $5 \%, 10 \%$ and $100 \%$ isomer dopants, respectively (Fig. 3). The isomer doping effect of RTA could be further corroborated since TCI-Cz and $0.5 \% \mathrm{Cc} / \mathrm{Lab}-\mathrm{Cz}$ in Fig. 3a, TCI-CPhCz and $0.5 \% \mathrm{CPhCc} / \mathrm{CPhCz}$ in Fig. 3e, TCI-DPhCzT and $0.5 \% \mathrm{DPhCcT} / \mathrm{DPhCzT}$ in Fig. 3i, respectively show nearly identical prompt and delayed spectra.

Fig. $3 b$, $f$ and $j$ show that the prompt emissions of $\mathrm{Cc} / \mathrm{Lab}-\mathrm{Cz}, \mathrm{CPhCc} / \mathrm{CPhCz}$ and $\mathrm{DPhCcT/DPhCzT}$ are red-shifted with increasing dopant content, which imply that Lab-Cz could benefit the development of deep-blue emitters as compared to those based on commercial $\mathrm{Cz}^{2}$. Moreover, the distinct differences (solid lines in Fig. $3 \mathrm{~b}, \mathrm{f}$ and $\mathrm{j}$ ) between the prompt emissions for each pair of isomers indicate that $\mathrm{Cz}$ and $\mathrm{Cc}$ own totally different electrondonating capabilities. In addition, the well-resolved fluorescence in Fig $3 \mathrm{~b}$ and $\mathrm{j}$ indicates the emissions are from the local-excited (LE) states ${ }^{27}$, while the broad fluorescence in Fig. $3 \mathrm{f}$ is the characteristic of charge-transfer (CT) emission ${ }^{19}$.

Fig. 3c shows the LE afterglow of Cc/Lab-Cz, with one band at 364-543 nm and the other at 543-836 nm. The component of shortwave afterglow varies with the dopant concentration, which agrees with the prompt emission (dashed lines in Fig 3b), indicating that the fluorescence of $\mathrm{Cc}$ is involved in afterglow. The intensity of long wavelength afterglow shows the highest at $1 \%$ doping. A similar phenomenon of LE afterglow is observed for DPhCcT/DPhCzT (Fig. 3k). For $\mathrm{CPhCc} / \mathrm{CPhCz}$ in Fig. 3g, the negligible $\mathrm{CT}$ afterglow and obvious $\mathrm{LE}$ afterglow with a maximum at $5 \%$ doping should be resulted from CT state to LE intersystem crossing ${ }^{26-28}$. These afterglow characteristics indicate that singlet and triplet excited states are simultaneously 
generated $^{12-14}$. Furthermore, the representative photographs of the doping effect with varying concentrations are shown in Fig. 3d, h and 1.
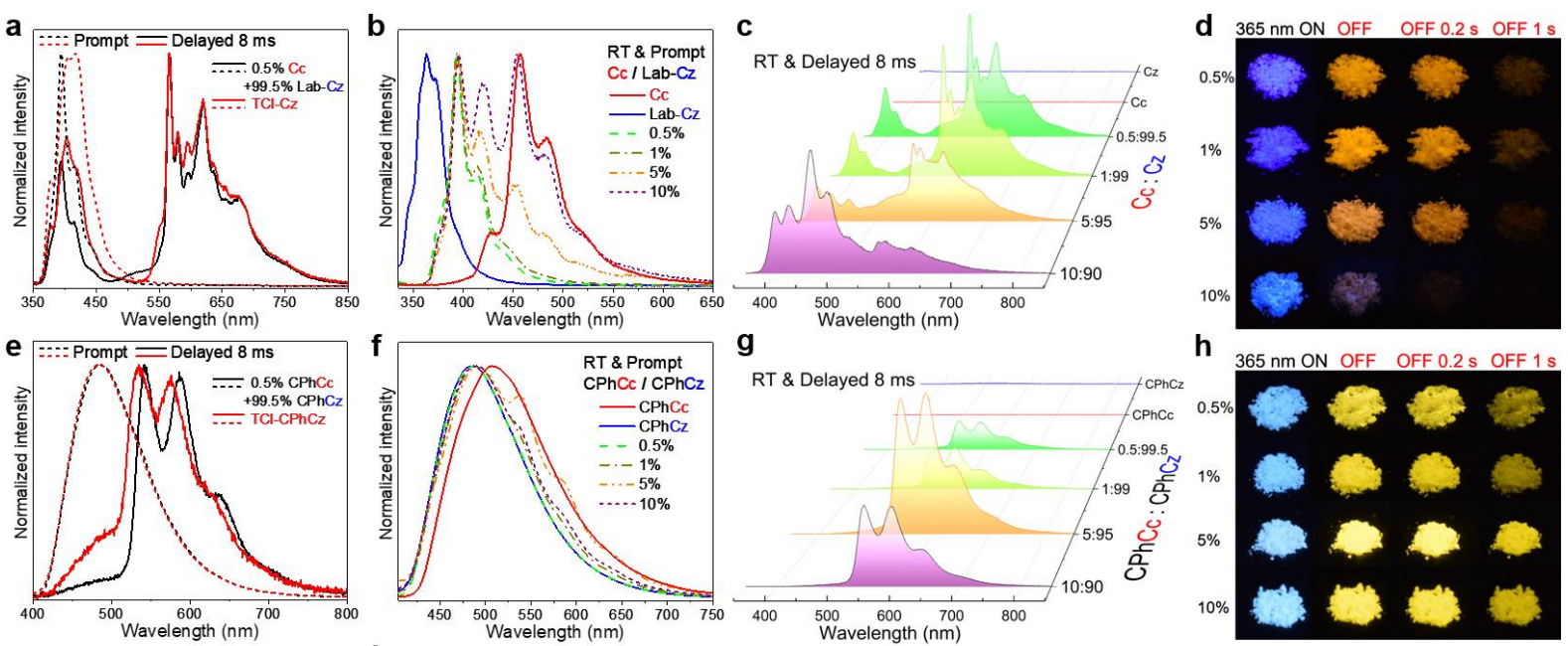

g

h $365 \mathrm{~nm}$ ON OFF OFF $0.2 \mathrm{~S}$ OFF 1 s
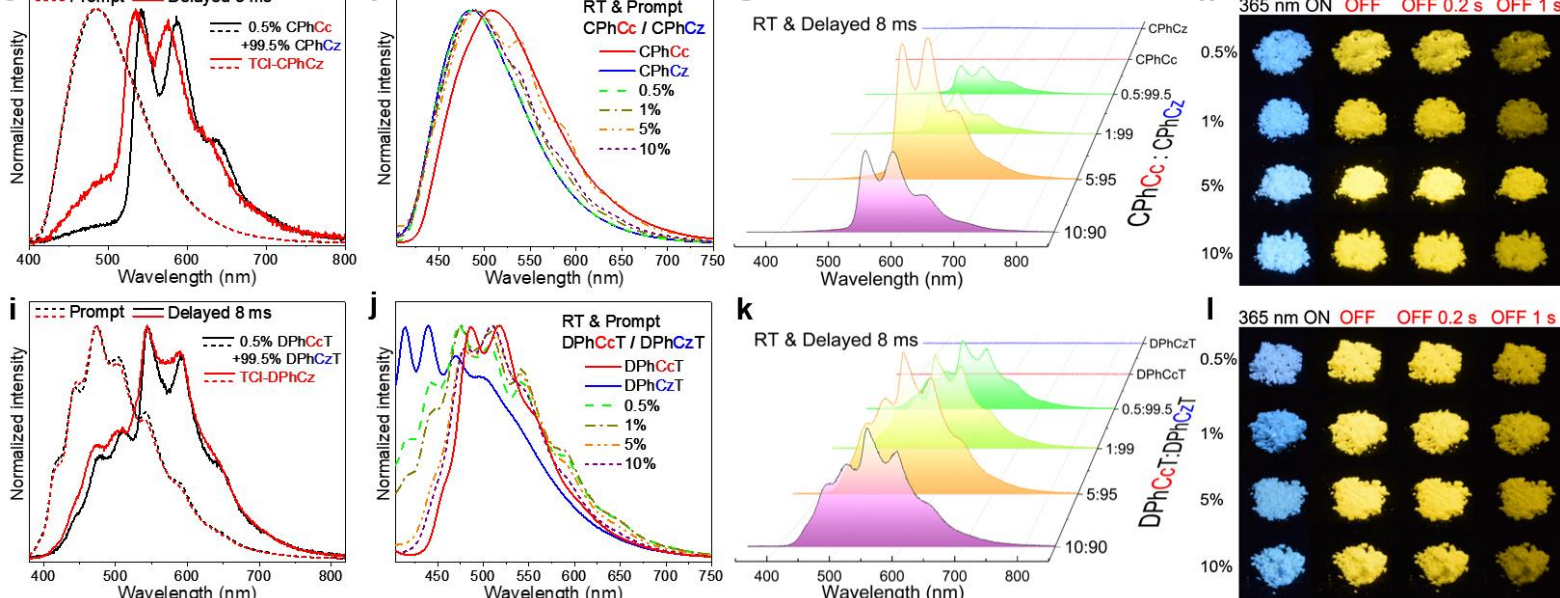

$\mathbf{k}$

I $365 \mathrm{~nm}$ ON OFF OFF $0.2 \mathrm{~s}$ OFF $1 \mathrm{~s}$

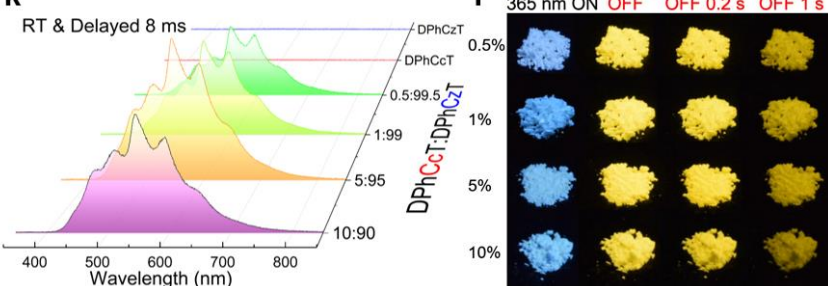

Fig. 3 | Photoluminescence characterizations with isomer dopants. a, e, i, PL spectra resolved into components of prompt and delayed $8 \mathrm{~ms}$ at ambient temperature in air, $0.5 \%$ $\mathrm{Cc} / \mathrm{Lab}-\mathrm{Cz}$ and TCI-Cz (a), 0.5\% CPhCc/CPhCz and TCI-CPhCz (e), 0.5\% DPhCcT/DPhCzT and TCI-DPhCzT (i). b, f, j, Prompt components of 0\%, 0.5\%, 1\%, 5\%, $10 \%$ and $100 \%$ isomer dopants; $\mathrm{Cc} / \mathrm{Lab}-\mathrm{Cz}(\mathbf{b}), \mathrm{CPhCc} / \mathrm{CPhCz}(\mathbf{f}), \mathrm{DPhCcT} / \mathrm{DPhCzT}(\mathbf{j})$. c, g, k, Delayed $8 \mathrm{~ms}$ components of $0 \%, 0.5 \%, 1 \%, 5 \%, 10 \%$ and $100 \%$ isomer dopants after $365 \mathrm{~nm}$ excitation off; $\mathrm{Cc} / \mathrm{Lab}-\mathrm{Cz}(\mathbf{c}), \mathrm{CPhCc} / \mathrm{CPhCz}(\mathbf{g}), \mathrm{DPhCcT} / \mathrm{DPhCzT}(\mathbf{k}) . \mathbf{d}, \mathbf{h}, \mathbf{l}$, Photographs of crystals with $0.5 \%, 1 \%, 5 \%$ and $10 \%$ isomer dopants of $\mathrm{Cc} / \mathrm{Cz}(\mathbf{d}), \mathrm{CPhCc} / \mathrm{CPhCz}(\mathbf{h})$ and $\mathrm{DPhCcT} / \mathrm{DPhCzT}$ (I). $310 \mathrm{~nm}$ excitation was used for the prompt spectra of $\mathbf{a}, \mathbf{b}$ and $365 \mathrm{~nm}$ excitation for all other measurements.

To elucidate the mechanism, transient absorption measurement with $8 \mathrm{~ms}$ integration time was conducted, so that the RTA and transient absorption spectra were synchronously recorded in 
Fig. 4a-c. The broad absorptions with peaks located at 460-475 $\mathrm{nm}$ are ascribed to radical ions, which is generated from charge separation ${ }^{12}$. Without doping, spectra at $8 \mathrm{~ms}$ traced noise after photo-activation (black lines in Fig.4a-c). While with doping, RTA and transient absorption of charge-separated states were captured (coloured lines in Fig. 4a-c) and showed the concomitant relation (Extended Data Fig. 8a).

Owing to the different electron-donating capabilities, $\mathrm{Cz}$ and $\mathrm{Cc}$ moieties in close proximity could act as a micro planar heterojunction to generate photoinduced charge-separated states ${ }^{12,26}$. RTA from $5 \%$ cross mixing systems of $\mathrm{Cc} / \mathrm{CPhCz}, \mathrm{Cc} / \mathrm{DPhCzT}, \mathrm{CPhCc} / \mathrm{Cz}$ and $\mathrm{DPhCcT} / \mathrm{Cz}$ (Fig. 4d and e) shows the emissions of newly generated charge-separated states (Extended Data Fig. 8b). Taking Cc/Cz as an example (Fig. 4f), two types of charge transfer could occur during photoexcitation. The generated $\mathrm{Cz}$ radical anions diffuse in the crystal ${ }^{5}$, forming chargeseparated states ${ }^{12,13}$. RTA is resulted from gradual charge recombination in the trap-detrap model of defects ${ }^{5,11-15}$. 


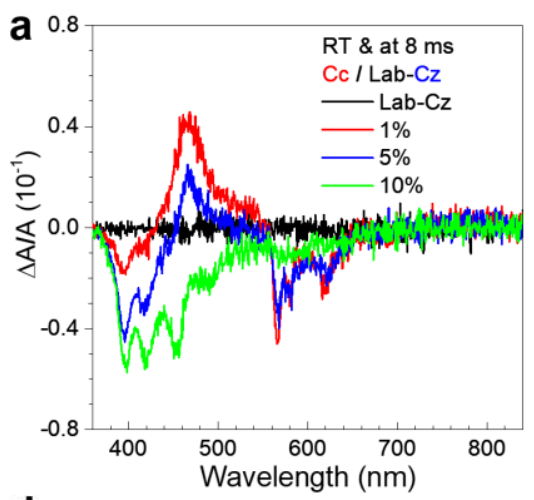

d

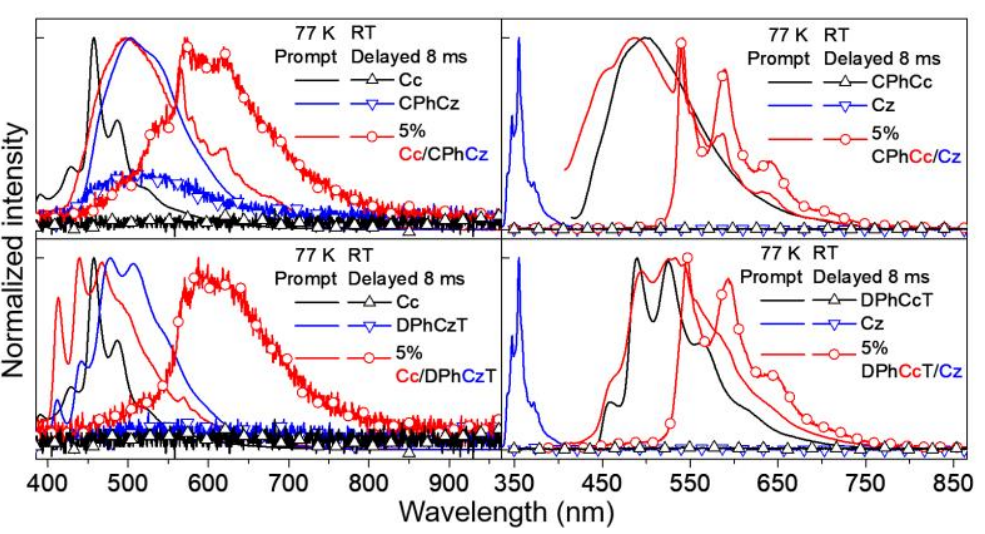

$\mathbf{f}_{\text {Charge transfer }}$

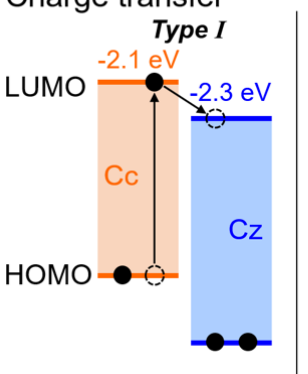

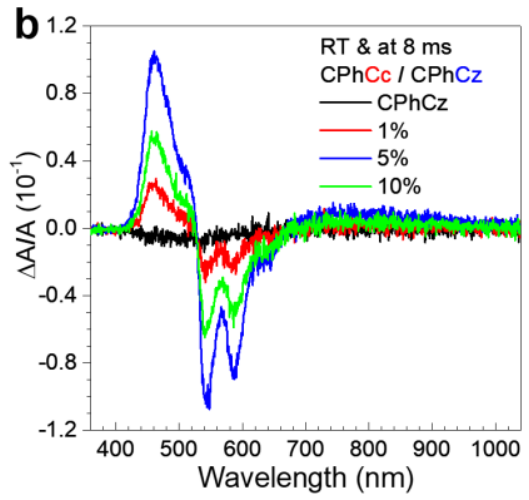

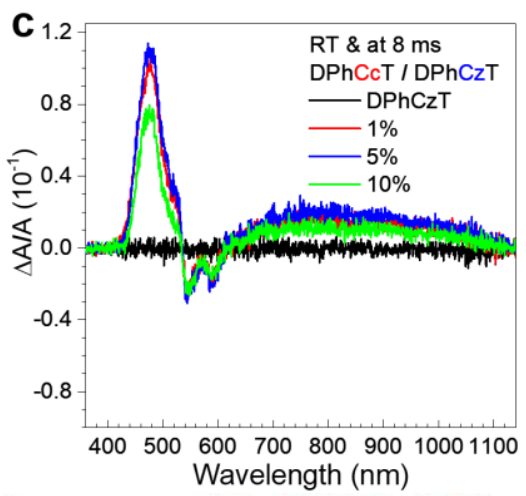

e $365 \mathrm{~nm}$ ON OFF OFF $0.2 \mathrm{~s}$ OFF $1 \mathrm{~s}$

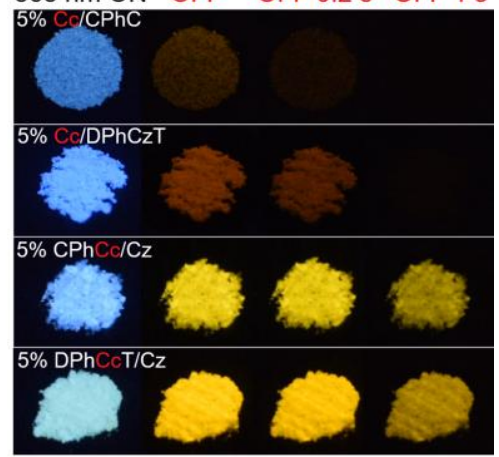

Charge recombination

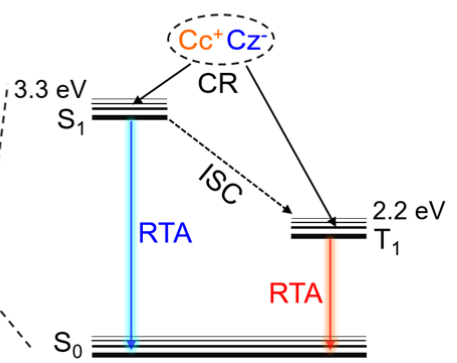

Fig. 4 | Photophysical properties and RTA mechanism. a, b, c, Photo-induced transient absorption of $0 \%, 1 \%, 5 \%$ and $10 \%$ isomer dopants; $\mathrm{Cc} / \mathrm{Lab}-\mathrm{Cz}(\mathbf{a}), \mathrm{CPhCc} / \mathrm{CPhCz}(\mathbf{b})$, $\mathrm{DPhCcT} / \mathrm{DPhCzT}(\mathbf{c})$. d, Emission of prompt at $77 \mathrm{~K}$ and delayed $8 \mathrm{~ms}$ at room temperature (RT) with cross mixing $5 \%$ doping systems of $\mathrm{Cc} / \mathrm{CPhCz}, \mathrm{Cc} / \mathrm{DPhCzT}, \mathrm{CPhCc} / \mathrm{Cz}$ and DPhCcT/Cz. e, Photographs of crystals under $365 \mathrm{~nm} \mathrm{ON,} \mathrm{OFF,} \mathrm{OFF} 0.2 \mathrm{~s}$ and OFF $1 \mathrm{~s}$. f, Proposed mechanism of RTA with $\mathrm{Cc} / \mathrm{Cz}$ as an example. Left, charge transfer during photoexcitation, Type I, electrons from the lowest unoccupied molecular orbital (LUMO) of $\mathrm{Cc}$ are transferred to the LUMO of Cz. Type II, electrons from the highest occupied molecular orbital (HOMO) of Cc are transferred to the HOMO of Cz. Middle, charge-separated states are formed with $\mathrm{Cz}$ radical anions diffusing to the neighbour $\mathrm{Cz}$, while $\mathrm{Cc}$ radical cations are trapped by the defects. Note that this intrinsic lattice defects may occur spontaneously during crystal growth. Right, singlets (e.g. $\mathrm{S}_{1}$ ) and triplets (e.g. $\mathrm{T}_{1}$ ) are generated from the charge recombination (CR), and intersystem crossing (ISC) of $\mathrm{S}_{1}$ to $\mathrm{T}_{1}$ is enabled. 
In summary, the comparison between lab-synthesized and commercial sources of $\mathrm{Cz}$, followed by optimization of HPLC with onset absorption offer a feasible solution to the impurity conundrums, which is applicable to other systems, such as commercial dibenzothiophene (Extended Data Fig. 9). Our studies elucidate that the impurity of $\mathrm{Cc}$, an isomer of $\mathrm{Cz}$, is responsible for the well-known RTA of $\mathrm{Cz}$. We have demonstrated that $\mathrm{Cc}$ and $\mathrm{Cz}$ are commensally derived within a series of $\mathrm{Cz}$ derivatives, which form the doping systems to activate their RTA. Owing to the similar reactivity, the widespread $\mathrm{Cc}$ in commercial $\mathrm{Cz}$ will trigger further investigations into other functional organic materials, wherein commercial $\mathrm{Cz}$ was fundamentally used in their syntheses. We are now isolating the impurities from commercial dibenzothiophene and dibenzofuran, which will extend new discoveries into more organic functional materials.

\section{References}

1. Uoyama, H., Goushi, K., Shizu, K., Nomura, H., Adachi, C. Highly efficient organic light-emitting diodes from delayed fluorescence. Nature 492, 234-238 (2012).

2. Hamze, R. et al. Eliminating nonradiative decay in $\mathrm{Cu}(\mathrm{I})$ emitters: $>99 \%$ quantum efficiency and microsecond lifetime. Science 363, 601-606 (2019).

3. Ai, X. et al. Efficient radical-based light-emitting diodes with doublet emission. Nature 563, 536540 (2018).

4. Sandanayaka, A. S. D. et al. Indication of current-injection lasing from an organic semiconductor. Appl. Phys. Express 12, 061010 (2019).

5. An, Z. et al. Stabilizing triplet excited states for ultralong organic phosphorescence. Nat. Mater. 14, 685-690 (2015).

6. Bilen, C. S., Harrison, N., Morantz, D. J. Unusual room temperature afterglow in some crystalline organic compounds. Nature 271, 235-237 (1978).

7. Clapp, D. B. The phosphorescence of tetraphenylmethane and certain related substances. J. Am. Chem. Soc. 61, 523-524 (1939).

8. Cai, S. et al. Visible-light-excited ultralong organic phosphorescence by manipulating intermolecular interactions. Adv. Mater. 29, 1701244 (2017).

9. Etherington, M. K. et al. Persistent dimer emission in thermally activated delayed fluorescence materials. J. Phys. Chem. C 123, 11109-11117 (2019).

10. Lastusaari, M. et al. The bologna stone: history's first persistent luminescent material. Eur. J. Mineral 24, 885-890 (2012).

11. Matsuzawa, T., Aoki, Y., Takeuchi, N., Murayama, Y. A new Long phosphorescent phosphor with high brightness, $\mathrm{SrAl}_{2} \mathrm{O}_{4}: \mathrm{Eu}^{2+}, \mathrm{Dy}^{3+}$. J. Electrochem. Soc. 143, 2670-2673 (1996).

12. Kabe, R., Adachi, C. Organic long persistent luminescence. Nature 550, 384-387 (2017).

13. Lin, Z., Kabe, R., Nishimura, N., Jinnai, K., Adachi, C. Organic long-persistent luminescence from a flexible and transparent doped polymer. Adv. Mater. 30, 1803713 (2018).

14. Jinnai, K., Kabe, R., Adachi, C. Wide-range tuning and enhancement of organic long-persistent luminescence using emitter dopants. Adv. Mater. 30, 1800365 (2018). 
15. Alam, P. et al. Two are better than one: A design principle for ultralong persistent luminescence of pure organics. (2019). doi:10.26434/chemrxiv.8298839.v1.

16. Xue, P. et al. Correction: Bright persistent luminescence from pure organic molecules through a moderate intermolecular heavy atom effect. Chem. Sci. 8, 6691-6691 (2017).

17. Graebe, C., Glaser, C. Ber. Dtsch. Chem. Ges. 5, 12 (1872).

18. Gu, L. et al. Dynamic ultralong organic phosphorescence by photoactivation. Angew. Chem. Int. Ed. 57, 8425-8431 (2018).

19. Mao, Z. et al. Two-photon-excited ultralong organic room temperature phosphorescence by dualchannel triplet harvesting. Chem. Sci. 10,7352-7357 (2019).

20. Xie, Y., Ge, Y., Peng, Q., Li, C., Li, Q., Li, Z. How the molecular packing affects the room temperature phosphorescence in pure organic compounds: ingenious molecular design, detailed crystal analysis, and rational theoretical calculations. Adv. Mater. 29, 1606829 (2017).

21. Xiong, Y. et al. Designing efficient and ultralong pure organic room-temperature phosphorescent materials by structural isomerism. Angew. Chem. Int. Ed. 57, 7997-8001 (2018).

22. Zhang, T., Wang, X., An, Z., Fang, Z., Zhang, Y., Yuan, W. Z. Pure organic persistent roomtemperature phosphorescence at both crystalline and amorphous states. ChemPhysChem 19, 23892396 (2018).

23. Yang, Z. et al. Intermolecular electronic coupling of organic units for efficient persistent roomtemperature phosphorescence. Angew. Chem. Int. Ed. 55, 2181-2185 (2016).

24. Kenry, Chen, C., Liu, B. Enhancing the performance of pure organic room-temperature phosphorescent luminophores. Nat. Commun. 10, 2111 (2019).

25. Zhao, W. et al. Boosting the efficiency of organic persistent room-temperature phosphorescence by intramolecular triplet-triplet energy transfer. Nat. Commun. 10, 1595 (2019).

26. Bolton, O., Lee, K., Kim, H.-J., Lin, K. Y., Kim, J. Activating efficient phosphorescence from purely organic materials by crystal design. Nat. Chem. 3, 205-210 (2011).

27. Chen, C. et al. Intramolecular charge transfer controls switching between room temperature phosphorescence and thermally activated delayed fluorescence. Angew. Chem. Int. Ed. 57, 1640716411 (2018).

28. Noda, H. et al. Critical role of intermediate electronic states for spin-flip processes in chargetransfer-type organic molecules with multiple donors and acceptors. Nat. Mater. doi.org/10.1038/s41563-019-0465-6 (2019).

Supplementary Information is available in the online version of the paper.

Acknowledgements This study was supported by the Singapore NRF Competitive Research Program (R279-000-483-281), NRF Investigatorship (R279-000-444-281) and National University of Singapore (R279-000-482-133). We thank G. K. Tan, L. K. Wong, Y. T. Pan, Dr. S. W. Zhou, Dr. Z. L. Xie, Dr. D. P. Ou, Prof. J. R. Xu and C. Y. Huang for technical assistance and discussions.

Author contributions C.C. and B.L. designed the experiments. C.C. optimized HPLC and grew crystals. C.C., Z.C., Z.Y., Z.M. and Z.Y. contributed to optical characterizations. C.C. and K.C. synthesized all compounds. A.S.B. and C.C. solved crystal structures. C.C. and B.L. discussed the results and edited the manuscript. B.L. supervised the project. 
Author information Reprints and permissions information is available at www.nature.com/reprints. The authors declare no competing financial interests. Correspondence and requests for materials should be addressed to B.L. (email: cheliub@nus.edu.sg).

\section{METHODS}

General. HPLC purifications were conducted by using XBridege ${ }^{\circledR}$ Prep C18 OBD $^{\mathrm{TM}} 5 \mu \mathrm{m}, 50 \mathrm{~mm} \times$ $150 \mathrm{~mm}$ column with $20.00 \mathrm{~mL} / \mathrm{min}$ based on Waters 2545 Binary Gradient Module, Waters 2707 Autosampler and Waters Fraction Collector III. HPLC injection volumn of Cz was $1500 \mu \mathrm{L}$ with 10.0 $\mathrm{mg} / \mathrm{mL}$, HPLC injection volumn of $\mathrm{CPhCz}$ was $1700 \mathrm{uL}$ with $4.0 \mathrm{mg} / \mathrm{mL}$. DPhCzT was not sufficiently soluble in methanol or ACN and hence it could not reveal the impurity peak by HPLC based on C18 column. Weights of the isolated impurities of $\mathrm{Cc}$ and $\mathrm{CPhCc}$ were determined by using differential weighing in their round bottom flasks. Therefore, we recorded as $\sim 10 \mathrm{mg}$ for $\mathrm{Cc}$ and $\sim 22 \mathrm{mg}$ for $\mathrm{CPhCc}$. NMR spectra were performed with a Bruker Avance-III 400 NanoBay HD NMR spectrometer at ambient temperature. High-resolution mass spectrometry (HRMS) was investigated with a Bruker AmaZon X LC-MS for electrospray ionization. X-ray diffraction experiments were carried out on a 4circle goniometer Kappa geometry Bruker D8 Venture diffractometer with a PHOTON 100 CMOS active pixel sensor detector. X-ray crystallographic data for structures reported here have been deposited in the Cambridge Crystallographic Data Centre (CCDC), under deposition numbers CCDC 19538021953811. These data can be obtained free of charge from The Cambridge Crystallographic Data Centre via www.ccdc.cam.ac.uk/data request/cif. The single crystals were grown by slow solvent evaporation at room temperature for 5-15 days ${ }^{27}$. Cz cystal was grown from ethanol and $\mathrm{Cc}$ crystal was grown from oxygen-free hexane in glovebox. $\mathrm{CPhCc}, \mathrm{CPhCz}$ and $\mathrm{DPhCzT}$ crystals were grown from dichloromethane (DCM)/hexane (1/1-1/3, v/v). To respectively compare TCI-Cz with Lab-Cz crystals, TCI-CPhCz with Lab-CPhCz crystals, and TCI-DPhCzT with Lab-DPhCzT crystals, we controlled each sets with the similar weights and volumes of solvent to grow crystals in the same scale vials at the same time, and then checked the crystals with microscope before X-ray diffraction. The reported afterglow disapeared in the single crystals of Lab set and appeared in the single crystals of TCI set. Xray diffraction experiments of impurities separated from TCI-Cz and TCI-CPhCz were investigated with the assistance of HRMS and NMR spectra. Cyclic voltammetry (Supplementary Information) was performed to calculate $\mathrm{HOMO}^{27}$ by BioLogic VMP-300 in a DCM $(99.9 \%$, Super Dry, stabilized, J\&K Seal) solution of $5 \times 10^{-4} \mathrm{M}$ sampe and $0.1 \mathrm{M} \mathrm{Bu}_{4} \mathrm{NPF}_{6}$ electrolyte. Electrodes: working (glassy carbon), counter (Pt disk), reference ( $\mathrm{Ag} / \mathrm{AgCl}$, calibrated against ferrocene), $100 \mathrm{mV} \cdot \mathrm{s}^{-1}$. Afterwards, LUMO was determined by ultraviolet/visible absorption with Hitachi U-3900. Purifications with silica gel column chromatography were performed using DAVISIL ${ }^{\circledR}$ silica LC60A 40-63 micro purchased from GRACE and monitored by TLC silica gel plates with 0.2-0.25 mm coating thickness from SANPONT. 
Cc isolated from TCI-Cz, ${ }^{1} \mathrm{H}$ NMR (400 MHz, $\left.\mathrm{CDCl}_{3}\right) \delta(\mathrm{ppm}): 8.07(\mathrm{~s}, 1 \mathrm{H}), 7.95(\mathrm{~s}, 1 \mathrm{H}), 7.88-7.80$ $(\mathrm{dd}, 2 \mathrm{H}), 7.73(\mathrm{~s}, 1 \mathrm{H}), 7.32-7.24(\mathrm{~m}, 3 \mathrm{H}), 6.60(\mathrm{~s}, 1 \mathrm{H}) ;{ }^{13} \mathrm{C} \mathrm{NMR}\left(101 \mathrm{MHz}, \mathrm{CDCl}_{3}\right) \delta(\mathrm{ppm}): 136.57$, 130.32, 129.61, 128.77, 128.27, 128.20, 127.30, 123.75, 122.61, 118.10, 106.22, 102.04; HRMS (ESI), $m / z:[\mathrm{M}+\mathrm{H}]$ 168.0805, calcd for $\mathrm{C}_{12} \mathrm{H}_{9} \mathrm{~N}, 167.0735$.

$\mathrm{CPhCc}$ isolated from TCI-CPhCz, ${ }^{1} \mathrm{H}$ NMR $\left(400 \mathrm{MHz}, \mathrm{CDCl}_{3}\right) \delta(\mathrm{ppm}): 8.88$ (s, 1H), 7.98-7.94 (q, 2H), 7.89-7.87 (t, 1H), 7.66-7.64 (d, 2H), 7.48-7.39 (m, 4H), 7.33-7.32 (d, 1H), 6.68-6.67 (d, 1H); ${ }^{13} \mathrm{C} \mathrm{NMR}$ $\left(101 \mathrm{MHz}, \mathrm{CDCl}_{3}\right) \delta(\mathrm{ppm}): 167.28,138.13,135.22,133.15,131.90,130.99,130.92,130.47,129.96$, 129.05, 128.81, 128.00, 125.19, 124.98, 118.89, 114.09, 109.29; HRMS (ESI), m/z: [M+Na] 328.0499, calcd for $\mathrm{C}_{19} \mathrm{H}_{12} \mathrm{ClNO}, 305.0607$.

DPhCcT synthesized from $\mathrm{Cc}^{5},{ }^{1} \mathrm{H}$ NMR $\left(400 \mathrm{MHz}, \mathrm{CDCl}_{3}\right) \delta(\mathrm{ppm}): 9.46(\mathrm{~s}, 1 \mathrm{H}), 8.73-8.72(\mathrm{~d}, 4 \mathrm{H})$, 8.61-8.60 (d, 1H), 8.06-8.04 (d, 2H), 7.92-7.90 (d, 1H), 7.59-7.57 (m, 6H), 7.47-7.39 (m, 2H), 6.87$6.86(\mathrm{~d}, 1 \mathrm{H}) ;{ }^{13} \mathrm{C}$ NMR $\left(101 \mathrm{MHz}, \mathrm{CDCl}_{3}\right) \delta(\mathrm{ppm}): 136.11,135.02,132.77,132.25,131.44,130.36$, 129.09, 128.84, 128.76, 128.00, 124.83, 124.55, 118.89, 114.54, 112.38, 108.69; HRMS (ESI), $m / z$ : $[\mathrm{M}+\mathrm{H}] 399.1602$, calcd for $\mathrm{C}_{27} \mathrm{H}_{18} \mathrm{~N}_{4}, 398.1531$.

Materials. Commerical carbazoles were obtained from TCI (Product of Japan, C0032-100 g), J\&K (Product of Beijing, 601413-250 g), Sigma-Aldrich (Product of Germany, C5132-100 g), and Aladdin (Product of Shanghai, C104875-100 g), and all were further recrystallized from toluene before using. After receiving from Arch Bioscience Company, Cc was further purified by column $(5 / 95, \mathrm{v} / \mathrm{v}$, EA/hexane) and then recrystallized from hexane. Light tan crystals were obtained ( $5 \%$ total purification yield), which has a different odor from Lab-Cz. Commercial dibenzothiophene was purchased from Sigma-Aldrich (Product of Belgium, D32202-25 g). Tetrahydrofuran (THF) was distilled with sodium and benzophenone. All solvents were HPLC grade from Fisher Chemical unless noted, and ultra pure water was produced by SMART2PURE of ThermoFisher Scientific. All other chemicals were obtained from commercial sources and directly used as received unless noted. $\mathrm{Lab}-\mathrm{Cz}$ was synthesized from 2-aminobiphenyl and lab-synthesized dibenzothiophene (Lab-DBT) was synthesized from 2-phenylthiophenol according to the literature ${ }^{29-30}$. CPhCz, DPhCzT, PhCz, CzDClT, CC4Br, C-C4-Br, $m$-MCBA, Cz-BP, BCz-BP and Cz-DPS were named and prepared according to their reported RTA references ${ }^{5,8,16,20-24}$. All of them were synthesized with both Lab-Cz and TCI-Cz, and then checked the purity with NMR. After confirming TCI-CPhCz was pure in NMR spectra, two more times of purification (1/2, DCM/hexane, v/v) by column were empolyed, and then further recrystallized from DCM/hexane solution. The obtained crystals were washed with fresh DCM/hexane (1/1, v/v) 3 times. RTA of TCI-CPhCz was continuously observed during the above purifications. To compare each pair of the products that synthesized from $\mathrm{Lab}-\mathrm{Cz}$ and TCI-Cz, respectively, the parallel conditions were controlled to do recrystallization. Afterwards, the same conditions (mentioned above) were controlled to grow single crystals for each pair of products. $\mathrm{CPhCc}$ and $\mathrm{DPhCcT}$ were synthesized under the same conditions of $\mathrm{CPhCz}$ and DPhCzT, respectively ${ }^{5,8}$.

All percentages for doping systems refer to mole percent in this paper. The samples were weighed by 
the METTLER TOLEDO balance with $0.01 \mathrm{mg}$ readability. $5 \%$ and $10 \%$ doping systems were prepared by weighing the samples and then disolved in organic solvent, THF/hexane $(2 / 1, \mathrm{v} / \mathrm{v})$ for $\mathrm{Cc} / \mathrm{Cz}$ systems, $\mathrm{DCM}$ for $\mathrm{CPhCc} / \mathrm{CPhCz}$ systems and $\mathrm{ACN}$ for DPhCcT/DPhCzT systems. $0.01 \%, 0.1 \%, 0.5 \%$, and $1 \%$ doping systems were prepared by dilution. For example, $2.000 \mathrm{~mL}$ was pipetted from $10.000 \mathrm{~mL}$ DCM solution of $3.28 \mathrm{mg} \mathrm{CPhCc}$ and $62.35 \mathrm{mg} \mathrm{Lab}-\mathrm{CPhCz}$. Then, $52.51 \mathrm{mg} \mathrm{Lab}-\mathrm{CPhCz}$ (calc. $52.47 \mathrm{mg}$ ) and $8.000 \mathrm{~mL}$ DCM were added to the above $2.000 \mathrm{~mL}$ solution to achieve $1 \%$ of $\mathrm{CPhCc} / \mathrm{CPhCz}$ solution. All solutions were slowly rota-vap at room temperature to get crystals. The $5 \%$ cross mixing systems were prepared by weighing the samples and then disolved in organic solvent, DCM for $\mathrm{Cc} / \mathrm{CPhCz}, \mathrm{ACN}$ for $\mathrm{Cc} / \mathrm{DPhCzT}$ and $\mathrm{THF} / \mathrm{hexane}(2 / 1, \mathrm{v} / \mathrm{v})$ for $\mathrm{CPhCc} / \mathrm{Cz}$ and $\mathrm{DPhCcT} / \mathrm{Cz}$.

Photographs. We used a handheld UV lamp of UVP UVGL-58 from Analytik Jena as the $365 \mathrm{~nm}$ irradiation source to take photographs at ambient temperature in air with Nikon D5300. $365 \mathrm{~nm}$ OFF is after $365 \mathrm{~nm}$ irradiation ceased $0.08 \mathrm{~s}$ and UV lamp is moved closer to excite samples before turning off. All $0.5 \%, 1 \%, 5 \%$ and $10 \%$ of $\mathrm{Cc} / \mathrm{Cz}, \mathrm{CPhCc} / \mathrm{CPhCz}$ and $\mathrm{DPhCcT} / \mathrm{DPhCzT}$ could be excited by white LED of iPhone6 and recorded by photographs in the Supplementary Information ${ }^{8}$.

Optical measurements. UV-visible absorption spectra of solution and solid were performed on Shimadzu UV-1700 and UV-3600 ultraviolet-visible-near-infrared (UV-Vis-NIR) spectrometers, respectively. Transient decay spectra, temperature-dependent PL spectra, decay curves and PL quantum yield (PLQY) were carried out using a Jobin Yvon-Horiba FL-3 spectrofluorometer and equipped with a calibrated integrating sphere. Notably, PLQY of these doping systems was randomly fluctuant, which was probably caused by the emission intensity changes with prolonging the photo-irradiation (Supplementary Information) ${ }^{18}$, and hence the PLQY was not reported here. Time-resolved RTA spectra (r.t. and $77 \mathrm{~K})^{19}$ and photo-induced transient absorption spectra (Supplementary Information) ${ }^{12}$ were obtained at ambient temperature in air through Ocean Optics QE65 Pro CCD with Ocean Optics LED-365 and LED-310 as excitation sources and Ocean Optics DH-2000-BAL as UV-VIS-NIR lightsource, which were assembled according to the references.

Data availability. The data that support the findings of this study are available from C.C. and L.B. on reasonable request.

29. Ullah, E., McNulty, J., Robertson, A. Highly chemoselective mono-Suzuki arylation reactions on all three dichlorobenzene isomers and applications development. Eur. J. Org. Chem. 2012, 2127-2131 (2012).

30. Nishino, K., Ogiwara, Y., Sakai, N. Green preparation of dibenzothiophene derivatives using 2-biphenylyl disulfides in the presence of molecular iodine and its application to dibenzoselenophene synthesis. Eur. J. Org. Chem. 2017, 5892-5895 (2017). 


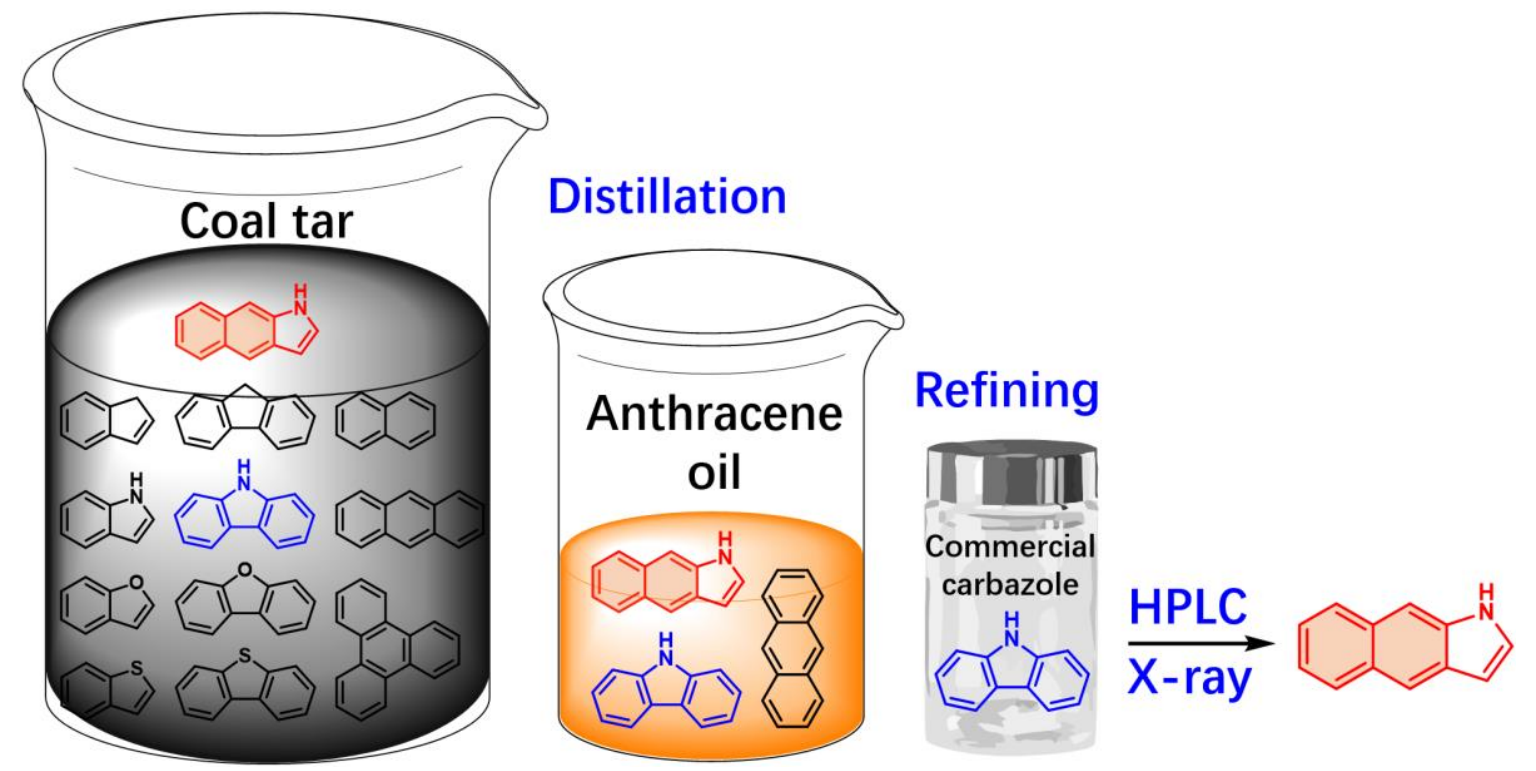

Extended Data Fig. 1 | Isolation process for commercial Cz. The isomer Cc is accompanyed.

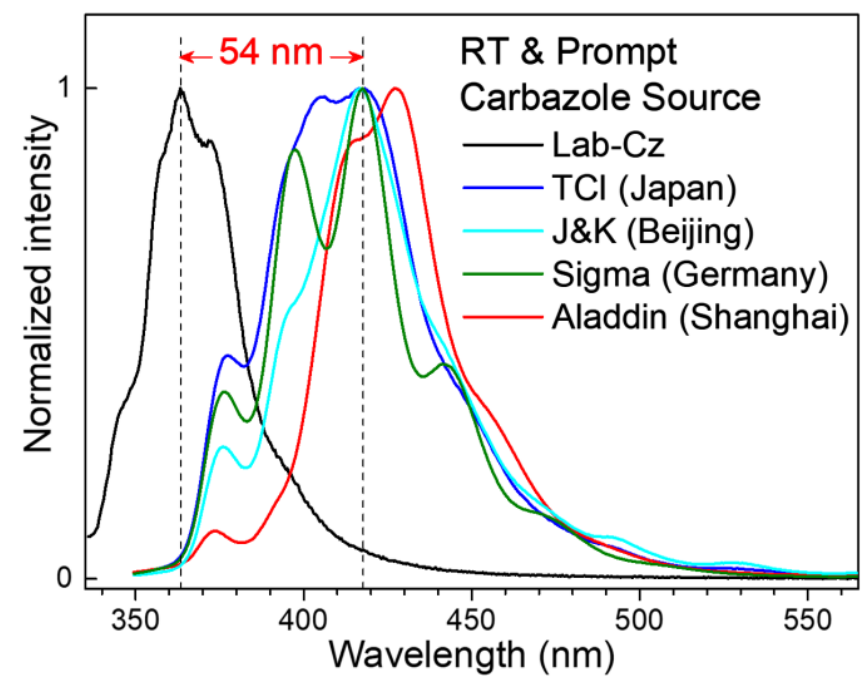

Extended Data Fig. 2 | Prompt spectra of $\mathbf{C z}$ from different sources. Fluorescence of the recrystallized Lab-Cz, TCI-Cz, J\&K-Cz, Sigma-Cz and Aladdin-Cz with $310 \mathrm{~nm}$ excitation. 

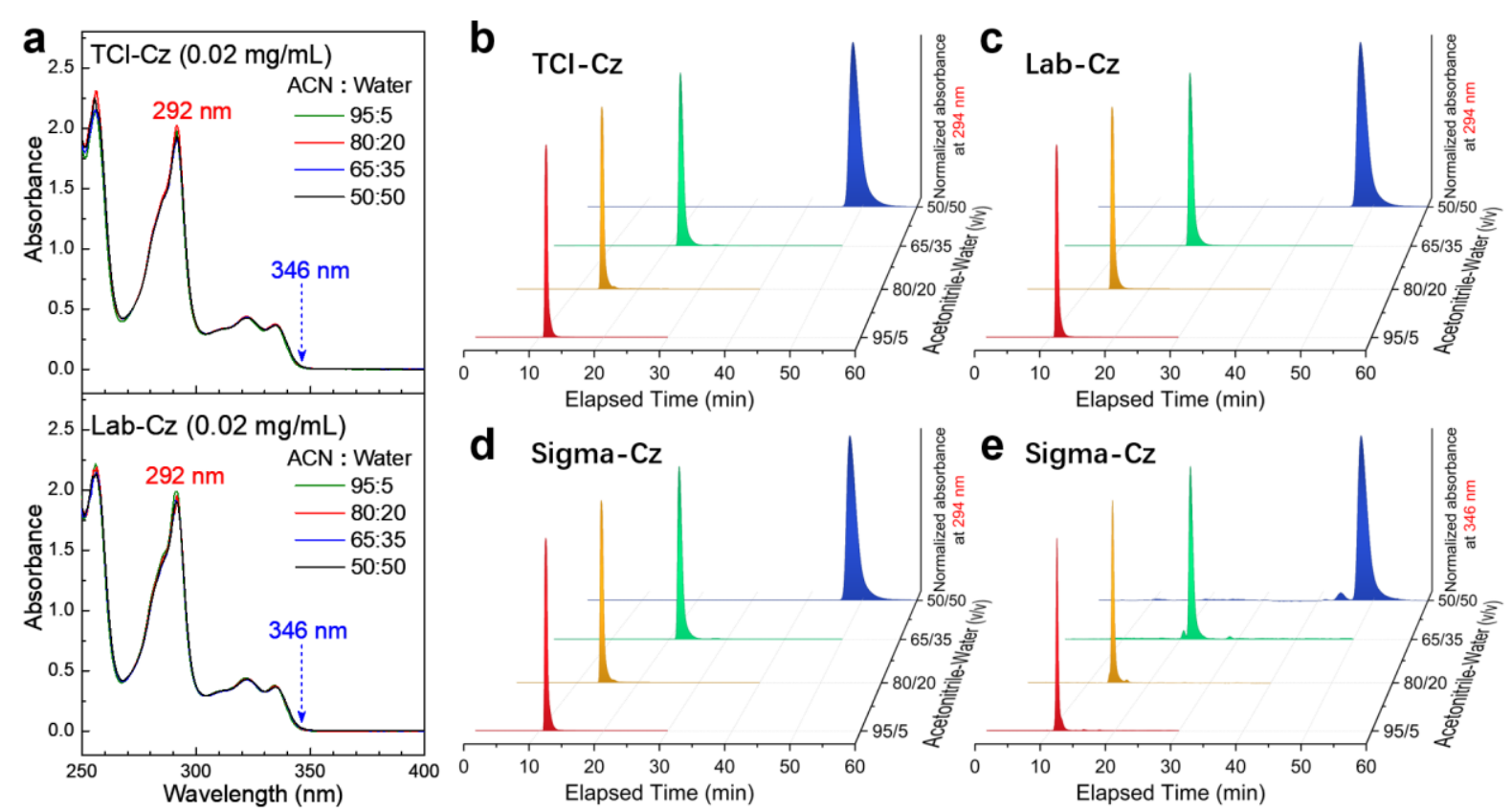

Extended Data Fig. 3 | Relationship between absorbance and HPLC optimization. a, Absorbance of TCI-Cz and Lab-Cz in solution with different volumn rates of acetonitrile-water. b, c, d, HPLC spectra monitored at $294 \mathrm{~nm}$ with optimizing acetonitrile-water ratio from 95/5 to 50/50 (v/v), TCI-Cz (b), Lab-Cz (c) and Sigma-Cz (d). e, HPLC spectra of Sigma-Cz monitored at $346 \mathrm{~nm}$ with optimizing acetonitrile-water ratio from 95/5 to 50/50 (v/v).
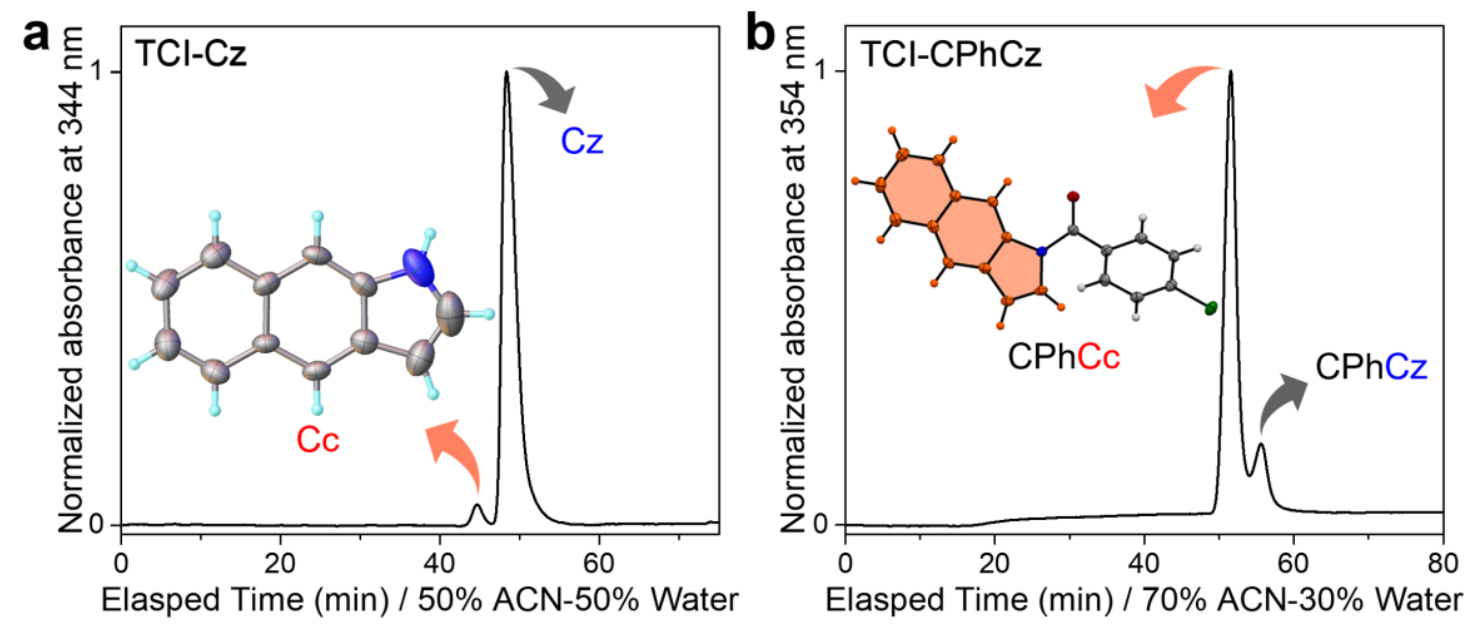

Extended Data Fig. 4 | X-ray crystallography based on HPLC purification. a, HPLC purificaion of commercial carbazole from TCI. b, HPLC purificaion of $\mathrm{CPhCz}$ synthesized from TCI-Cz, and we used 5\% DCM/hexane (v/v) for a chromatographic column to concentrate the impurity before HPLC. Single crystals were grown from the HPLC purified components and their structures were determined by X-ray diffraction. 


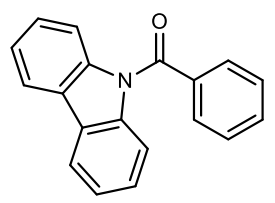

$\mathrm{PhCz}$<smiles>Clc1nc(Cl)nc(-n2c3ccccc3c3ccccc32)n1</smiles>

CzDCIT

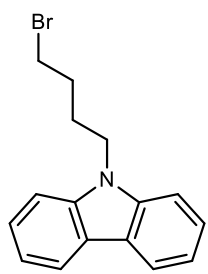

$\mathrm{CC} 4 \mathrm{Br}$
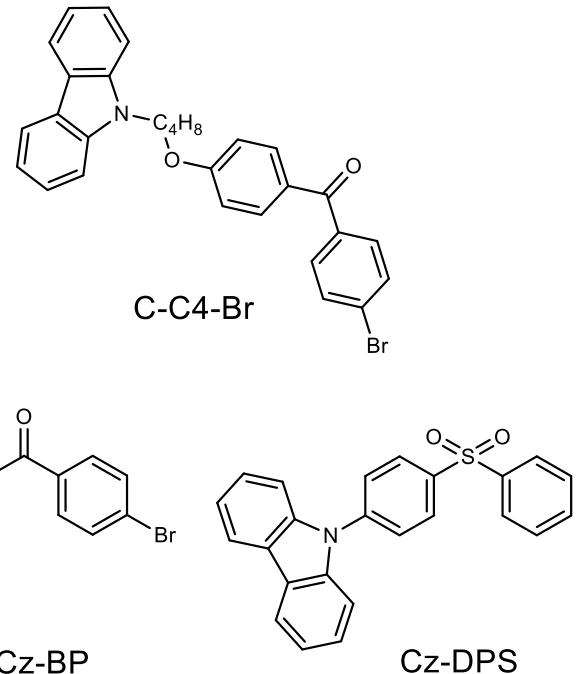

Cz-DPS

$m$-MCBA<smiles>O=C(c1ccccc1)c1ccc(-n2c3ccccc3c3ccccc32)cc1</smiles><smiles>O=C(c1ccc(Br)cc1)c1ccc(-n2c3ccccc3c3ccccc32)cc1</smiles>

$\mathrm{BCz}-\mathrm{BP}$

Extended Data Fig. 5 | RTA paradox of $\mathrm{Cz}$ derivatives from $\mathrm{Lab}-\mathrm{Cz}$ and TCI-Cz.

Chemical structures of reported and representative RTA molecules ${ }^{5,8,16,20-24}$. Their reported RTA was only observed with the crystals of products synthesized from TCI-Cz, but not from Lab-Cz.
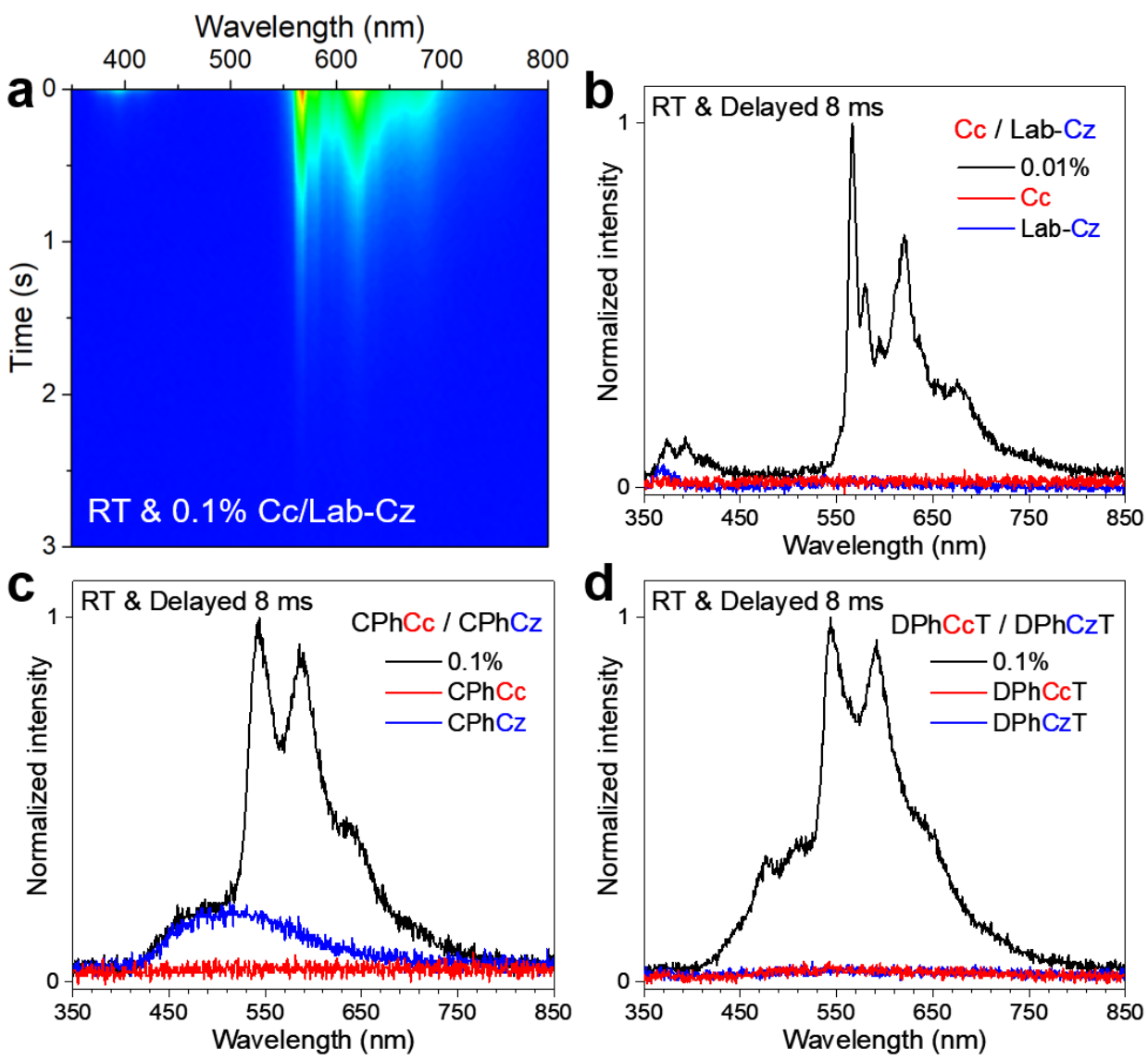

Extended Data Fig. 6 | Photoluminescence characterizations of isomer doping systems. a,

Time-resolved RTA spectra of $0.1 \% \mathrm{Cc} / \mathrm{Lab}-\mathrm{Cz}$ crystals, excited at $365 \mathrm{~nm}$ and integral time $8 \mathrm{~ms}$. b, c, d, Normalized PL spectra of crystals at $8 \mathrm{~ms}$ after $365 \mathrm{~nm}$ excitation ceased, $0.01 \%$ $\mathrm{Cc} / \mathrm{Lab}-\mathrm{Cz}(\mathbf{b}), 0.1 \% \mathrm{CPhCc} / \mathrm{CPhCz}(\mathbf{c})$ and $0.1 \% \mathrm{DPhCcT} / \mathrm{DPhCzT}$ (d). 
a

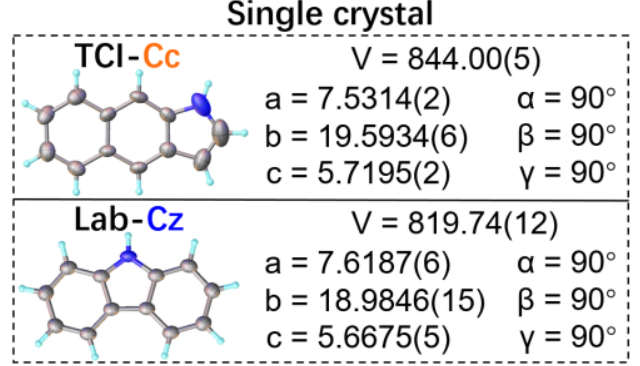

b

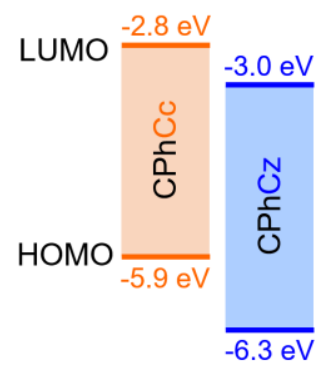

C

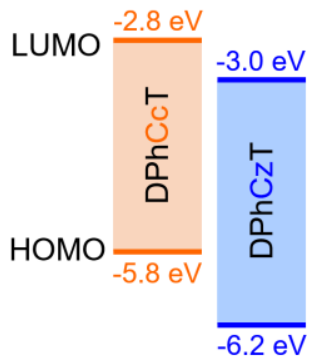

Extended Data Fig. 7 | Isomers. a, Single crystal structures with unit cell parameters of Cc (separated from TCI carbazole, TCI-Cc) and Cz (synthesized from 2-aminobiphenyl, Lab-Cz). b, c, HOMO and LUMO values of $\mathrm{CPhCc}$ and $\mathrm{CPhCz}(\mathbf{b}), \mathrm{DPhCcT}$ and $\mathrm{DPhCzT}$ (c) from the cyclic voltammetry and absorption spectra.
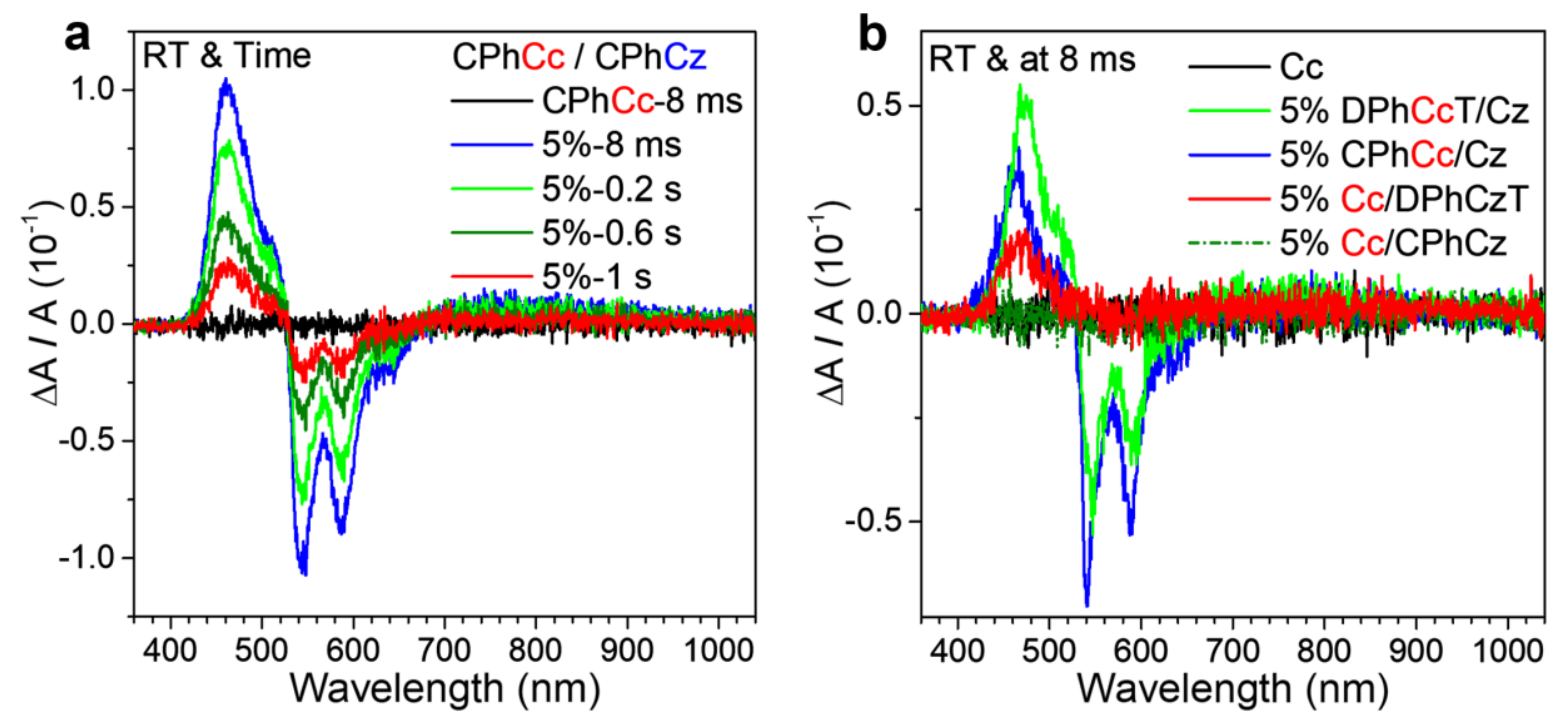

Extended Data Fig. 8 | Photo-induced charge separation. a, Photo-induced transient absorption of $5 \% \mathrm{CPhCc} / \mathrm{CPhCz}$ crystals at different time, and compare with that of the nondoped $\mathrm{CPhCc}$ crystals at $8 \mathrm{~ms}$. b. Photo-induced transient absorption of 5\% $\mathrm{DPhCcT} / \mathrm{Cz}$, $\mathrm{CPhCc} / \mathrm{Cz}, \mathrm{Cc} / \mathrm{DPhCzT}$ and $\mathrm{Cc} / \mathrm{CPhCz}$ crystals at $8 \mathrm{~ms}$, and compare with that of the nondoped Cc crystals at $8 \mathrm{~ms}$. 


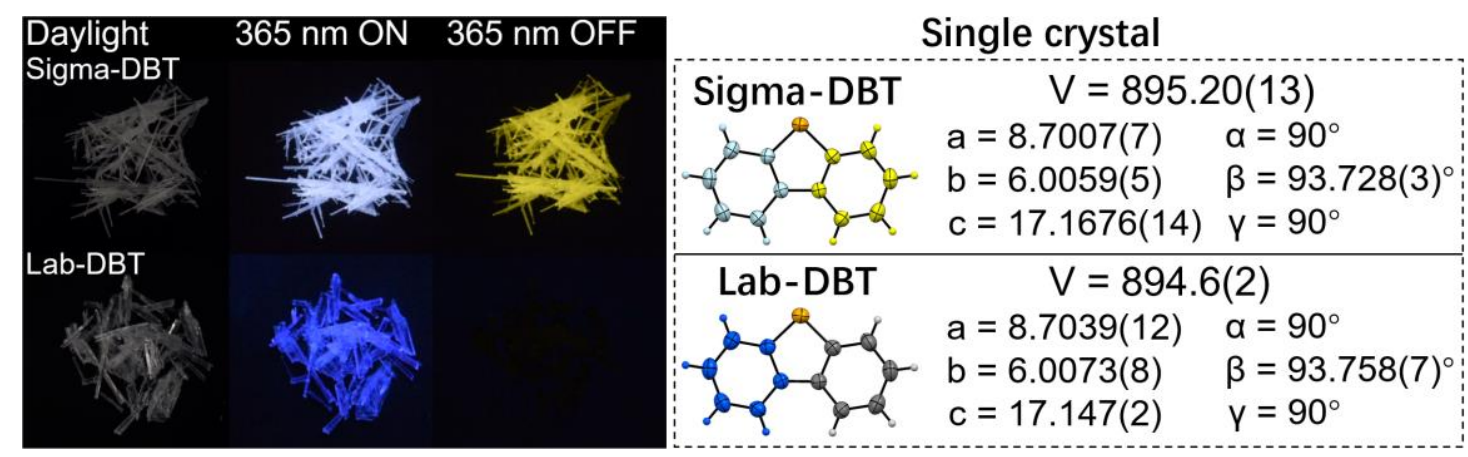

Extended Data Fig. 9 | RTA paradox of Sigma-DBT and Lab-DBT. Crystal photographs of dibenzothiophene from Sigma-Aldrich (Sigma-DBT) and dibenzothiophene synthesized from 2-phenylthiophenol (Lab-DBT) at daylight, $365 \mathrm{~nm}$ irradiation ON/OFF, and their single crystal structures with unit cell parameters. 